\title{
TOXICITY EVALUATION OF A NOVEL RAPAMYCIN LIPOSOMAL FORMULATION AFTER SUBCONJUNCTIVAL AND INTRAVITREAL INJECTION
}

\author{
Rodrigo Garcia-Santisteban ${ }^{1}$, María Josefa Bernad-Bernad ${ }^{2}$, Pedro Javier Salas Ambrosio ${ }^{2}$, \\ Monica Linares-Alba ${ }^{2}$, Luis Tonix-Aburto², Guadalupe Ornelas-Lobato ${ }^{2}$, Isabel \\ Gracia-Mora $^{2}$, Marisol Rivera-Huerta ${ }^{2}$, Francisco Sánchez-Bartez ${ }^{2}$, Héctor Rico-Morales ${ }^{2}$, \\ and Gustavo García-Sánchez ${ }^{2}$ \\ ${ }^{1}$ Universidad Panamericana - Campus México \\ ${ }^{2}$ Universidad Nacional Autonoma de Mexico
}

September 21, 2020

\begin{abstract}
Purpose: Safety and toxicity evaluation of a nove liposome-encapsulated rapamycin formulation, intended for autoimmune ocular disorders. Methods: The formulation (60 and $180 \mu \mathrm{g}$ for subconjunctival and 40 and $440 \mu \mathrm{g}$ for intravitreal administration) were assessed through micronucleus polychromatic erythrocytes production, and irritability by HET CAM and pyrogenicity testing. Subconjunctival and intravitreal administration of the formulation were performed to evaluate subacute and acute toxicity, respectively. Shapiro Wilk and Kolmogorov-Smirnov tests were applied for normality. Pearson and Spearman Correlation tests for variable comparison. Differences between groups in biochemical and hematological parameters were evaluated by ANOVA test with significance determination by t-Holm-Sidak post-hoc test. A t-test was employed to compare independent, normally distributed variables. Numeric score was assigned to histopathological classification. Data was analyzed by a one way no parametric Kruskal-Wallis and the Mann-Whitney tests. Significance was considered when p $<0.05$ Results: No significant toxicity directly related to the preparation was detected. Micronucleus count, mucous irritation score, and pyrogenicity were negative. Pathology demonstrated no damage related to the formulation after subconjunctival injection. After intravitreal injection, only lens injury associated with the technique was observed. Retinal function was also conserved in electroretinography. (ERG). Conclusions: The preparation evaluated offers a good toxicity and safety profile when injected in a subconjunctival or intravitreal manner in an animal model. A clinical trial conducted in humans is highly warranted, as it could reveal an alternative immunosuppressive treatment for ophthalmological immune-mediated pathologies.
\end{abstract}

\section{INTRODUCTION}

Rapamycin, also called sirolimus, is a macrolide with antifungal, antineoplastic, and immunosuppressive properties discovered in 1975 (1). It was approved by the United States Food and Drug Administration in 1999 for the prophylaxis of organ rejection in human patients older than 13 years at a dose of $2 \mathrm{mg} /$ day $(2-3)$. It is practically insoluble in water $(2.6 \mu \mathrm{g} / \mathrm{mL})$, yields high liposolubility (log PO/W 5.77), very unstable in ionic medium and has demonstrated a high rate of degradation under UV light (4-6). Despite its liposolubility, the drug has low corneal penetration, and has therefore not been able to show a high effectivity rate for the treatment of autoimmune ocular surface disorders (7).

Some of the advantages of rapamycin as an immunosuppressant derive from its unique mechanism of action, its improved side-effect profile, and its ability to synergize with other immunosuppressive agents (calcineurin inhibitors) due to the shared metabolism by CYP 3A (8). Because of this, its potential clinical uses range 
from organ transplantation to ocular disorders, including non-infectious uveitis, diabetic macular edema, autoimmune non-necrotizing anterior sclerosis and Sjögren syndrome (9-12)

Rapamycin pharmacokinetics display large inter- and intra-patient variability, due to different disease stages and use of concurrent immunosuppressants or other interacting drugs, as well as polymorphisms in the genetic configuration of CYP 3A. Due to the long half-life of rapamycin, dose adjustments should ideally be based on levels obtained 5-7 days after initiation of therapy or dosage change (13). Unfortunately, although rapamycin-induced toxicity has been reported extensively in patients with organ rejection, there are few reports concerning specific ocular toxicity. Adult horse and rabbit models with different ophthalmologic disorders have been used for this purpose. Administration through intravitreal (IVT) and subconjunctival (SCJ) injection at doses ranging from $20 \mu \mathrm{g}$ to $10 \mathrm{mg}$ have reported no evidence of tissue damage or toxicity (14-15). The safety profile of rapamycin in adult human patients has also been evaluated, no toxicity was reported at doses ranging from 44 to $1760 \mu \mathrm{g}$. (10-11)

Carriers can be used to enhance the intraocular concentrations of drugs, as well as minimize the dose, and therefore side effects of medications. One type of such carriers are liposomes. Liposomes are microscopic vesicles composed of lipid bilayers surrounding an aqueous core (16-17). They can improve the solubilization and absorption of a lipophilic drug encapsulated inside the vesicle, as well as the bioavailability in the ocular environment while protecting it from degradation (18-20). Since liposomes have a similar composition as cell membranes, they are expected to be biocompatible and biodegradable (21). Furthermore, due to the fact that liposomes release constant low doses of drugs instead of the usual single complete dose in one take, they are expected to be able to reduce nonspecific side-effects and toxicity. (22). Nevertheless, the thorough study of the toxicologic profile of new pharmaceutical formulations is always important in the drug development process. Taking into consideration the scarcity of in vivo studies demonstrating the assumed non-toxicity of liposomes prepared through non-conventional solvent free methodologies only tested in vitro in non-ocular cell lines by other authors (23), the aim of this study is to describe the results of an in vitro and in vivo toxicity evaluation of liposomes loaded with rapamycin using a proprietary methodology, administered through subconjunctival and intravitreal injections to support their potential as a treatment of ocular inflammatory autoimmune disorders.

\section{MATERIALS AND METHODS}

\subsection{Materials}

Rapamycin loaded liposomes (RL) and placebo liposomes (L) were provided by Laboratorio Santgar (Mexico City, MX), and produced using a proprietary methodology (23-24). Test formulations were kept under refrigeration $\left(2^{\circ} \mathrm{C}-8^{\circ} \mathrm{C}\right)$ at the experimental facility until use.

For the micronucleus test, male Hsd:ICR Specific Pathogen Free (SPF) certified mice were purchased from UNAM-ENVIGO Center of Laboratory Animal Production (Coyoacan, MX). Light microscopy DM2500 (Leica Biosystems, Nussloch, DE) was used for micronucleus determinations as well as slide observation in the subacute toxicity study.

For the HET-CAM assay, 9 day-old SPF hen's embryos were purchased from ALPES, S.A. de C.V. (Puebla, MX). Sodium dodecyl sulfate (SDS) from Sigma-Aldrich (Saint Louis, USA) was used as HET-CAM positive control. Physiologic saline solution (PhS) purchased from PISA, S.A. de C.V. (Guadalajara, MX) was used as a negative control in this study as well as in the Intravitreal acute retinal toxicity study (IARTS) and the subacute toxicity study (STS).

For pyrogenicity assay and STS, healthy certified male New Zealand rabbits were purchased from Science Animals (Mexico City, MX). Independently, 3 animals were purchased for pyrogenicity assay and 12 animals for STS. Rectal temperature measurements were done using a Sejoy MT 401 (Hangzhou Sejoy, CN) calibrated rectal thermometer for pyrogenicity assessment.

SCJ injections in STS as well as intravitreal injections in IARTS were performed using new, 30G needles, attached to $1 \mathrm{ml}$ sterile disposable syringes (Becton Dickinson and Co., New Jersey, USA). Proparacaine HCl 
0.5\% veterinary ophthalmic solution (Paracainaß) provided by Laboratorio Santgar (Mexico City, MX) was used as local anesthetic in STS and IARTS. For STS, blood samples were collected using both empty Microtainer@ and Microtainer@ containing EDTA K2. (Becton Dickinson and Co., New Jersey, USA). Hematologic samples were processed in a BCVet analyzer (KONTROLAB International Corp., Rome, IT). Hematological slides were stained using a semiautomatic system Hematek (R) (Siemens Healthcare GmbH, Munich, DE). Biochemical analysis was performed by the automated chemistry analyzer CST-240 (DIRUI Industrial Co., Ltd., Changchun, CN).

For IARTS, 15 healthy certified New Zealand rabbits were purchased from Soluciones MG (Mexico City, MX). Fundus photographs were recorded using a Kowa Genesis-D Handheld Retinal Camera (Kowa American Corporation, Torrance, USA), Cyclopentolate 1\%, Phenilephrine $\mathrm{HCl} \mathrm{2.5 \%}$ and Tropicamide $1 \%$

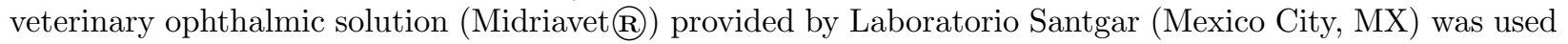
as local mydriatic. Electroretinograms were recorded with a BMP 200 Retinographics electrodiagnostic system (Dioptrix, Touluse, FR). For IVT injections, animals were anesthetized with isoflurane Sofloran Vet $\mathbb{R}$. (PiSA Agropecuaria, S.A. de C.V. Tula, MX) and sodium pentobarbital Sedalpharmaß) (Pet's Pharma, Nezahualcoyotl, MX). In all cases, animal euthanasia was induced with an overdose of sodium pentobarbital Pisabental@ (PiSA Agropecuaria, S.A. de C.V. Tula, MX) according to local normativity.

\subsubsection{Liposome preparation:}

The preparation of the proprietary liposomes has been previously described (24-25). The liposomes were composed of lecithin and cholesterol, preparation included dilution by injection of ethanol. Stirring at 450-500 $\mathrm{rpm}$ for about $40 \mathrm{~min}$ was performed. Afterwards, the formulation was heated to $45^{\circ} \mathrm{C}$ and then stirred at 350 rpm until the ethanol was completely evaporated. Sterilization was done aseptically through a polyvinylidene difluoride (PVDF) hydrophilic membrane with a $\mathrm{N}_{2}$ pressure inlet of 2 bars and then refrigerated. Afterwards, the lipid dispertions were hydrated with prepared Phosphate buffered saline preparation and reheated at $70^{\circ} \mathrm{C}$ while stirring at $750 \mathrm{rpm}$ for $30 \mathrm{~min}$. The mixture was then left at room temperature for 30 minutes with N2. Required sirolimus was then added. The dispersion was filtered through a hydrophilic membrane and collected in refrigerated glass.

This method of sirolimus liposomes preparation is proprietary to the team and has an FDA-issued patent. It is of critical importance to highlight the differences between this formulation and the one used by other investigators (26). First of all, our liposomes do not employ the thin lipid film hydration method, we also do not use chloroform in the mixture, and do not need to place the tube under vaccum for 12 hours. Furthermore, our formulation does not use a buffer containing $6 \%$ trehalose. Finally, the concentration used by these authors is of $5 \mathrm{mg} / \mathrm{ml}$, while the concentration employed by us ranges from $0.4-1 \mathrm{mg} / \mathrm{ml}$.

\subsection{Methods}

All the following tests were conducted under a Quality Management System that assures the accomplishment of Good Laboratory Practices (GLP). The experimental facility, Preclinical Research Center of National Autonomous University of Mexico (UNIPREC-UNAM) had registration at the EMA/OCDE (BPL-002/15) accreditation unit, for GLPs endorsement. Also, the center was approved by local Mexican authority (SENASICA AUT-B-B-0919-056) for animal experimentation.

Experimental protocols were approved by the Institutional Committee for the Care and Use of Laboratory Animals (CICUAL) of the UNAM Faculty of Chemistry. All animals were treated in accordance with local and international guidelines followed by CICUAL for the ethical use of laboratory animals in research.

Care for animals used in this research is in concordance with the Association for Research in Vision and Ophthalmology (ARVO) statement for the Use of Animals in Ophthalmic and Vision Research.

\subsection{Evaluation of Genotoxicity by in vivo micronucleus test}

The animal model and sample size were selected according to OECD guidelines for Mammalian Erithrocyte Micronucleus Test (27). Fifteen male mice Hsd:ICR Specific Pathogen Free, weighing between 25-30 g were 
randomized into three groups: 1) positive control using cyclophosphamide, 2) RL and 3) L. $40 \mathrm{ml} / \mathrm{kg}$ doses were administered through intraperitoneal injection. Posterior to this, three blood samples were taken from caudal vein as follows: before the administration, $36 \mathrm{~h}$ and $72 \mathrm{~h}$ after administration. Samples were fixed with ethanol at $70 \%$ for 10 minutes, stained with Giemsa $10 \%$ solution and observed with light microscopy by a trained specialist. Quantification of polychromatic erythrocytes for each thousand erythrocytes and micronucleated polychromatic erythrocytes (MPE) for each two thousand polychromatic erythrocytes were determined for each sample time.

Results were analyzed by two ways-ANOVA with a post Hoc Tukey test. A p value of $<0.05$ was considered significant using Sigmaplot software version 13.

2.4. HET CAM mucous irritation potential analysis.

Mucous irritation potential of a drug can be evaluated by observing its effects on the chorioallantoic membrane of a fertilized, incubated hen's egg. This method is considered by some to be an alternative to the Draize eye irritation test (28). 10 day-old Specific Pathogen Free chicken embryos were used. On the $10^{\text {th }}$ day, eggshells were carefully removed, and the exposed membranes were inoculated in triplicate with $300 \mu \mathrm{L}$ of SDS $1 \%$ for positive control, Phs $0.9 \%$ for negative control, and RL \& L for test product. Afterwards, for the following 5 minutes after inoculation, membranes were monitored for hemorrhage, vascular lysis and coagulation reaction. Time to each reaction was recorded in seconds. The Irritation Score was calculated according to DB-ALM:INVITTOXX protocol No. 96, as follows:

$$
\text { IS }=[((301-\mathrm{sec})(\mathrm{H})(0.5))+((301-\mathrm{sec})(\mathrm{L})(0.7)+((301-\mathrm{sec})(\mathrm{C})(0.9)] /(300 * 300 * 300)
$$

Where H: hemorrhage; L: vascular lysis; C: coagulation; sec: initial time in seconds. Assignation to severe grades on each reaction were: $0=$ no reaction, $1=$ low, $2=$ moderate and $3=$ severe reaction.

\subsection{Determination of Pyrogenicity}

The increase in temperature due to pyrogens was analyzed in vivo. The test was performed according to compendial methodology described in the Pharmacopoeia of the United Mexican States (FEUM) (29). Test dilutions (1:10) were prepared by diluting test formulations with Phs $0.9 \%$ in aseptic conditions. $1.5 \mathrm{~mL}$ were administrated intravenously in the marginal ear vein of three healthy male New Zealand rabbits weighting 1.5 to $3.0 \mathrm{~kg}$. Rectal temperature was measured employing a digital thermometer. Basal temperature was recorded in the normal range $\left(38-39.8^{\circ} \mathrm{C}\right)$ prior to administration. Post administration measurements were recorded each $30 \mathrm{~min}$ for 3 hours. In case no subject presented an increase in temperature of more than 0.6 ${ }^{\circ} \mathrm{C}$ from its basal temperature, and the sum of the global increments did not exceed $1.4{ }^{\circ} \mathrm{C}$, the sample was considered pyrogen free.

2.6. Evaluation of subacute toxicity in vivo in male New Zealand rabbits after subconjunctival injection.

We performed a set of in vitro toxicity tests that will be later discussed, in order to warrant in vivo experimentation. A subacute toxicity study was performed according with US Food and Drug Administration (FDA) Center for Veterinary Medicine Guidance for Industry \#185 (CVM GFI \#185) (28) with some modifications which scientific rationale will be later discussed.

Sample size was calculated using G-power software 3.1 version (Heinrich-Heine-Universität Düsseldorf, DE) with a power of $80 \%$. Twelve young male adult New Zealand rabbits weighting $2.0 \mathrm{~kg}(+/-0.5 \mathrm{~kg})$ received subconjunctival rapamycin doses, empty liposomes and injectable water as control in groups of 3 rabbits each (Table1). Doses were administrated once weekly for three weeks $(0,1,7$ and 14 days). First, anesthesia was performed by instilling two drops of proparacaine $0.5 \%$ in the ocular surface of the rabbits. One minute after instillation, bulbar dorsal conjunctiva was slightly raised and the corresponding preparation was slowly injected at the superior fornix until formation of a bulge was observed in the inner conjunctiva.

Ophthalmologic examination was performed after each injection by trained veterinarians using slit-lamp and fundoscopy. Changes in gait, posture and behavior were also monitored daily by trained personnel in the 
bioterium of the University (UNAM) by releasing them from enclosure and using food to encourage the animals to walk.

\subsubsection{Assesment of metabolic changes by weight monitoring}

Body weight of each subject was recorded at 1, 7, 14 and 21 days. Interaction between days and groups, comparisons between groups, initial and final weight were analyzed with the ANOVA test $(p<0.05)$.

\subsubsection{Biochemical assay}

Blood samples were collected from marginal vein before treatment and on the $10^{\text {th }}$ and $22^{\text {nd }}$ days of followup. Previously, subjects were fasted for 4-6 hours to reduce postprandial biochemical changes and stress. For hematologic tests, $400 \mu \mathrm{L}$ were collected in Microtainers $\mathbb{R}$ containing EDTA as anticoagulant and for biochemical assays, $700 \mu \mathrm{L}$ were collected in empty Microtainers@. Non-anticoagulated blood samples were centrifuged after blood clot was formed to obtain blood serum. Samples were analyzed using an automated chemistry analyzer to observe any renal or hepatic alterations caused by formulation treatment. Serum glucose (GL) levels, urea, creatinine (CR), cholesterol (CH), total bilirubin (TB), alkaline phosphatase (AP), alanine aminotransferase (ALAT), aspartate aminotransferase (ASAT), calcium, phosphorus (Ph), total proteins and albumin were measured.

\subsubsection{Hematology tests}

Hematological parameters evaluated included hematocrit (Hat), hemoglobin (Ham), erythrocyte count (Ert), mean corpuscular volume (MCV), mean corpuscular hemoglobin (MCH), and total leucocytes (Leu). Determinations were made using an automated hematological analyzer. Subsequently, blood smears were performed and examined through the microscope for white blood cell differential (WBCD), platelet estimate, and Ert morphologic examination.

\subsubsection{Necropsy}

On days 21 and 22, animals were euthanized through intravenous anesthetic overdose. All rabbits were submitted to a complete necropsy. Samples of the following tissues were collected for histopathology: Brain, liver, kidney, parotid and mandibular salivary glands, right and left eyes, optic nerve, eyelids (lower and upper), internal and external tear glands, as well as two lymph nodes, one maxillary and one retropharyngeal.

Samples were placed in $10 \%$ buffered formalin, dehydrated and embedded in paraffin. Afterwards, 3-micron thick slices were obtained. Slices were stained with hematoxylin and eosin (H\&E) and Gram stain for further microscopic examination by a veterinary pathologist.

2.7. Evaluation of acute retinal toxicity in vivo in New Zealand rabbits after intravitreal injection.

In order to assess the potential retinal toxicity of rapamycin liposomes, an evaluation of retinal function and histology was performed after administration of two doses (40 and $440 \mu \mathrm{g})$ of liposomal rapamycin applied through intravitreal injection.

Sample size was calculated using the same parameters as the subconjunctival injection group. As a result, 15 young adult New Zealand rabbits weighting $2.0 \mathrm{~kg}(+/-0.5 \mathrm{~kg})$ were selected to receive intravitreal injections of liposomes loaded with rapamycin, empty liposomes and Phs as negative control (Table 2). The injection procedure was conducted as follows: Anesthesia was induced by inhalation of isoflurane according to standard protocol of UNIPREC-UNAM, also a drop of topical anesthetic was applied. After one minute, 0.1 $\mathrm{mL}$ of the test product was injected through a $30 \mathrm{G}$ needle $2 \mathrm{~mm}$ posterior to the limbus directed towards the center of the vitreous cavity. Povidone-iodine solution was instilled to prevent microbial infection. Finally, an ophthalmologic evaluation was performed to detect immediate adverse reactions. An ophthalmologic evaluation was conducted every 24 hours for 14 days by trained veterinarians using slit lamp and fundoscopy. Gait and behavioral changes were monitored by trained personnel in the bioterium releasing the animals from enclosure and using food as bait to evaluate gait.

2.7.1. Electroretinography and fundus pictures 
Basal electroretinographic measurements and fundus pictures were registered prior to injection procedures. First, mydriasis was induced with topical tropicamide. After a dark adaptation period of 1 hour and topical anesthesia, an ERG of each eye was recorded. The active electrode with golden ring was placed on the cornea, the reference electrode was introduced subcutaneously near the lateral canthus and the ground electrode subcutaneously in the back of the neck, scotopic flash electroretinogram was recorded. 7 days after the experiment, a second ERG was recorded, and new fundus pictures were taken.

\subsubsection{Histopathology}

On day 14, animals were euthanized by intravenous anesthetic overdose. In the postmortem study, a macroscopic evaluation of the ocular structures was executed and those with evidence of pathology were considered for microscopical exam. Ocular globes were collected for histopathology, including optic nerve and corneas.

Samples were fixed in 10\% buffered formalin, dehydrated and embedded in paraffin, then, 3-5 micron thick slices were obtained. Slices were stained with hematoxylin and eosin $(\mathrm{H} \& \mathrm{E})$ for further microscopic examination by a veterinary pathologist.

\subsection{Statistical Analysis}

Shapiro Wilk and Kolmogorov-Smirnov test were applied to all results to verify if they were normally distributed. Pearson or Spearman Correlation test were carried out to determine if variables or individuals were similar. Differences between groups in biochemical and hematological parameters was evaluated by ANOVA test with significance determination by t-Holm-Sidak post-hoc test. A t-test was employed to compare 1:1 independent normally distributed variable. For histopathologic comparisons in SCJ toxicity study, due to qualitative nature of the data, a numeric score was assigned for further comparison. Data was analyzed by a one way no parametric Kruskal-Wallis test for multiple group comparison and MannWhitney test was employed to compare 1:1 independent variable. For retina IVT toxicity, correlation between histopathologic findings and treatment was All results were evaluated at $p<0.05$ using SigmaStat@ version 13.0 software. Plots were edited by Origin Lab 2016® or Microsoft Excel 365®.

\section{RESULTS}

3.1. Evaluation of genotoxicity by in vivo micronucleus test (MPE).

According to Figure 1, there was no increase of MPE in groups treated with rapamycin liposomal formulation or empty liposomes compared with positive control, where there was an evident increase of MPE at 36 hours.

3.2. HET CAM mucous irritation potential analysis.

Irritation score of the formulations and control were $<0.9$, Table 3.and thus corresponds to no reaction according to standarized guidelines (27), while positive control showed a moderate reaction. These results were observed in egg-chorioallantoic membranes, Figure 2, which showed increased membrane vascular lysis, hemorrhage and coagulation when SDS was placed in contact, in contrast with RL and L formulations that had no effect.

\subsection{Determination of pyrogenicity}

According to the following exclusion criteria, established in the Mexican Pharmacopeia FEUM (29), pyrogen absence is warranted if:

a) No rabbit temperatures differ more than $2^{\circ} \mathrm{C}$ between two temperatures consecutively measured at the acclimatization period

b) No rabbit temperature is higher than $39.8^{\circ} \mathrm{C}$ or lower than $38.0^{\circ} \mathrm{C}$

c) No rabbit temperature increases individually above $0.5^{\circ} \mathrm{C}$ compared to basal temperature during the test

d) No rabbit shows an increase in temperature above $0.6{ }^{\circ} \mathrm{C}$ compared to its individual basal temperature, and the sum of the maximum increment observed in the 3 rabbits does not exceed $1.4{ }^{\circ} \mathrm{C}$. 
Rapamycin loaded liposomal formulations met the mentioned requirements and can therefore be considered pyrogen free (Table 4).

3.4. Subacute toxicity in vivo in male New Zealand rabbits after subconjunctival injection.

\subsubsection{Metabolic changes}

No deaths or apparent adverse clinical signs were found in any group throughout the study period. There was no statistical difference in mean body weights in the comparisons made between groups. As it is shown in the results presented in Table 5, there was no discrepancy.

\subsubsection{Clinical evaluation and histopathologic analysis}

All the specimens were observed macroscopically at least twice a day through the entire duration of the study. Upon examination, they presented adequate general body condition. Mucous membranes had typical pale pink color and good state of preservation; however, in all groups lacrimal glands reached two times their normal size. This effect can probably be attributed to a transitory parasympathetic stimulation effect caused by the injection on the lacrimal gland processed by afferent fibers in the conjunctiva and sclera of the eye. This is supported by the fact that the effect was seen in both treatment and control groups and by that after the second subconjunctival injection, rabbits \#1, 2 and 11 presented tearing that resolved within 2 days. Rabbit \#1 also presented swollen eyelids and pain, which also resolved within 2 days post administration. After euthanasia of the specimens, a complete necropsy was performed. The internal general inspection detected mild to moderate pulmonary, hepatic and renal congestion in all groups. Figure 4 presents some of the observed alterations after histopathological analysis of the samples. Histopathological observations are summarized in Figure 5, $p$ values of group comparison for each lesion demonstrate that no statistically significant differences between groups, including controls, were found.

\subsubsection{Biochemical assay}

Results are summarized in Table 6. No toxicity-indicative differences were found between groups, sampling time or interaction between group and sampling time in all experiments.

\subsubsection{Hematologic testing}

Results are summarized in Table 7. no relevant differences between groups were noted in sampling time or interaction group-sampling time.

3.5. Evaluation of acute retinal toxicity in vivo in New Zealand rabbits after intravitreal injection.

All the specimens were observed macroscopically at least once a day through the entire duration of the study by trained veterinary ophthalmologists. Upon examination, they presented adequate general condition although mild ocular and/or palpebral irritation was observed in test groups but not in control group. Fundus pictures showed no evident macroscopical alterations in retinal structure, Figure 6 presents representative pictures of basal appearance versus post injection aspect of retinas.

\subsubsection{Electroretinography}

No reduction in amplitude, increased implicit time or alteration in waveform was observed between the basal measurements and post injection response or in tests groups versus control group. U Mann-Whitney test $(\mathrm{p}<0.05)$ was performed to determine significance. Implicit times of $\mathrm{b}$-wave measured in test groups compared against control group response 7 days after treatment are presented in Figure 7, statistical analysis revealed no changes associated to test products.

Representative ERG waveforms of each group 7 days after IVT injection are presented in Figure 8. Statistical difference was tested between b-wave amplitude 7 days after treatment compared with the basal measurement of each group (Fig.8). No significant detriment in amplitude was observed in any group, the group administered with $40 \mu \mathrm{g}$ of rapamycin resulted in statistically different amplitude due to an increase in amplitude after IVT injection. Amplitude detected 7 days post injection of test groups was also compared 
with that of the control group, Figure 9. As well as comparative results versus basal measurements, only the group administered with $40 \mu \mathrm{g}$ of rapamycin was statistically different from control group due to increased amplitude observed in this group after treatment.

\subsubsection{Histopathology}

No evident pathological alterations such as detachment or hemorrhages were found in the macroscopic observation of retinas. Nevertheless, some specimens showed signs of traumatic lens fragmentation. No other alterations were observed in the rest of the ocular structures. Figure 10A shows microscopic findings. No histopathological injury was found in the control group or the group treated with $40 \mu \mathrm{g}$ of rapamycin. In the rest of the groups, retinal degeneration and hyperplasia was observed at least in one sample. In order to determine the significance of this findings, a one way-nonparametric Kruskal-Wallis ANOVA analysis was performed with significance at $\mathrm{p}<0.05$, Fig. 10B. However, no significant differences were found between groups. Representative histologic micrographs of microscopic observations are shown in Figure 11.

\section{DISCUSSION}

4.1. Evaluation of genotoxicity by in vivo micronucleus test.

Increased cancer risk is a serious adverse effect among patients undergoing immunosuppressive therapy. Micronucleus frequency has been reported to be significantly higher in pediatric patients with immunosuppressive therapy after kidney transplant (30). Although the dose challenged in this paper is topical and much lower, this novel RL formulation is being proposed for a chronic disease that may imply long term therapy, which is why assessment of genetic damage is mandatory.

The results presented in Figure 1 indicate a lack of genotoxicity effect, which is consistent with studies reporting that unlike other immunosuppressants, when used specifically for ocular inflammation, rapamycin has been reported to inhibit immunosuppression-induced neoplasia (31). This could be explained by the different mechanism of action of rapamycin versus other immunosuppressants. Oncogenic transformation, one of the most worrying consequences of DNA damage, is favored by the loss of cell cycle control and the activation of growth promoting pathways, such as the signaling path involving the mammalian Target of Rapamycin (mTOR). Rapamycin and other inhibitors of mTOR decelerate cell proliferation and contribute to avoid oncogenic transformation by suppressing the signals required for cell cycle progression, cell growth and proliferation (32). Thus, RL as well as its liposomal vehicle, are not expected to produce genetic damage when administered in mammals.

\subsection{Evaluation of mucous irritability potential by HET CAM analysis}

HET CAM test is an in vitro alternative to the in vivoDraize test, which is one of the most criticized methods of ocular irritation testing because of the injuries inflicted on test animals. The occurrence of vascular injury or coagulation in egg-chorioallantoic membranes in response to a compound, has a good correlation with the Draize rabbit test (33). This test provides information about immediate effects after administration of a product, mainly by vascular alterations, but also by protein interactions making it an adequate pre-screen method of eye injury hazard potential. The results indicate that the product is not expected to produce irritation effects on ocular membrane when tested in vivo. The obtained results were expected, taking into consideration previous reports of lack of ocular toxicity of rapamycin in animals. However, testing of the vehicle irritation potential and the combination with rapamycin was yet to be proved. These results justify the innocuity of the product in order to be tested in live animals, as well as confirms biocompatibility of the selected nanocarrier.

\subsection{Determination of pyrogenicity}

Pyrogens are substances that can produce fever when present as contaminants in a drug. Most pyrogens are biological substances derived from microorganisms that trigger an immune response, they can be lifethreatening to patients because the produced systemic reaction can go from fever to neurologic effects, shock and death. These results are of great importance since some of the most recognized drawbacks for the 
application of liposomal technology in medicine are presence of organic solvent residues, difficult pyrogen control and sterility assurance, poor stability and adequate size distribution. It is worth mentioning that the formulations were also tested for sterility with good compliance with FEUM standards (data not published), along with absence of pyrogenic reaction. This means that proprietary methodology used for the preparation of the samples tested in this work is suitable for commercial production.

Aside of ruling out endotoxin contamination, absence of pyrogenicity gives indirect information on acceptable size distribution of RL, since it has been reported that liposomes larger than $200 \mathrm{~nm}$ tend to cause non-endotoxin-dependent rise in temperature, probably due to the metabolism of lipid mediators like prostaglandins (34). Lack of pyrogenic reaction suggests a suitable size distribution of the RL liposomes for intraocular injection.

4.4. Subacute toxicity in vivo in male New Zealand rabbits after subconjunctival injection.

Subacute systemic toxicity is defined as the adverse effects occurring after multiple or continuous exposure between $24 \mathrm{~h}$ and 28 days. For this kind of studies, the FDA recommends testing the maximum dose intended for therapeutic use, and 3 and 5 times that dose to identify effects associated with overdosing and increased duration of administration (35). Also, in this early stage of our development process it will help to establish a safe dose range for future optimal dose exploration.

In this study the dose range tested was limited to only 3 times the dose intended for therapeutic use and the administration frequency was doubled. Rationale for these modifications rely on the limitations of the route of administration. The maximum volume generally recognized as safe for subconjunctival injection (SCJ) is $0.5 \mathrm{ml}$, for this study the highest dose was rounded to $0.45 \mathrm{ml}$ that represents 3 times the therapeutic dose. At first, we tried to inject $0.45 \mathrm{ml}$ divided in 2 injections of $0.225 \mathrm{ml}$, considering that an injection volume of $0.2 \mathrm{ml}$ is commonly used in practice with no remarkable adverse effects. However, significant discomfort was observed in the rabbits when manipulated for the second injection, apparently due to the stress caused by prolonged manipulation. Ultimately, it was decided to perform a single injection for subsequent administrations. Regarding administration frequency, instead of testing a higher dose, weekly administration was established in the protocol, that frequency is twice as frequent as the dose regimen considered for therapeutic use.

\subsubsection{Metabolic changes}

No difference in mean body weights in the comparisons between treatment groups was found. Although comparisons between final and initial weight show a slight alteration was observed, it is attributable to the innate growth of the rabbits. Hence, no significant metabolic changes were noted.

\subsubsection{Clinical evaluation and histopathologic analysis}

Macroscopic evaluation only revealed mild alterations in 3 subjects after the second injection. Necropsy detected discrete pulmonary, renal and hepatic congestion in all test and control animals probably related to the euthanasia method. Other organs did not present relevant disturbances. The microscopic examination of histologic slices after necropsy revealed odd pathology results for each tissue, (Figure 5A). For both right and left eyes, lymphoplasmacytic uveitis was detected mainly in control subjects. Due to these alterations, especially in rabbit \#1, all the slices were stained with Gram. One subject (rabbit 1, group 3) was detected with presence of Encephalitozoon cuniculi spores, (Figure 4B). This subject exhibited multifocal lymphoplasmacytic infiltrates in stroma and ciliary body of the right eye, this finding was also detected in right lacrimal internal gland, as well as mild multifocal necrosis. The left eye of this specimen showed similar injury, but the infiltration covered also the iris structure. In the left lens slice multiple spores of Encephalitozoon cuniculiwere found, as well as in liver and kidney samples. Optical nerve samples of this subject presented mild multifocal lymphoplasmacytic meningitis.

Based on macroscopic and microscopic findings, it was determined that 6 subjects $(2$ rabbits from group 2, 3 rabbits from group 3 and 1 rabbit from group 4) presented injuries compatible with encephalitozoonosis but histopathological confirmation of spores was only possible in rabbit \#1 from group 3. Most of the injuries 
observed were similar between groups, this statement was confirmed by statistical analysis that displayed no significant differences among treatment groups so no relationship between treatment and organic damage could be elucidated. Figure 5 summarizes the injuries observed in the microscopic examination of the subjects. It can be inferred that the highest injury levels were presented in group 3 treated with liposomal vehicle. The decreased quantity and level of injuries detected in animals administered with rapamycin containing formulations compared with empty liposomes may be related to the local immunosuppressant and anti-inflammatory activity of rapamycin (36). Lymph nodes in all groups (Figure 5A) exhibited lymphoid hyperplasia at mild multifocal level, these can be appreciated in Figure 4D where germinal centers of the lymph node present discrete hyperplasia. Also, as mentioned, all the rabbits showed lymphoplasmacytic dacryoadenitis. These findings are consistent with the presence of Encephalitozoon infection (37).

Histologic examination of the liver detected hepatocellular degeneration in all groups, Figure 5A. Group 1 showed only mild hepatocellular degeneration and congestion. Furthermore, subjects of groups 2,3 and 4 presented moderate periportal lymphoplasmacytic hepatitis, Figure 4E. Possibly, hepatitis could be associated with lymphoid activity and hepatocellular degeneration increase and necrosis could be related toEncephalitozoon cuniculi infection, Figure $4 \mathrm{~F}$, since these lesions were found near the parasite spores in rabbit \#1 (38). It is difficult to attribute these injuries to the administration of the test product since the most affected rabbits were in the control and vehicle groups. Also, it was not possible to determine a statistically significant difference between treatment groups. Mild to moderate multifocal tubular degeneration was observed in the kidneys of all groups. Additionally, lymphocytic interstitial nephritis in groups 2 and 3 was presented with one subject at level 4 and other at level 7, which is represented in Figure 4G. Both, degeneration and nephritis could be a consequence of the parasitic infection. No trend was observed in kidney injuries between groups due to rapamycin administration [37].

Lymphocytic encephalitis and satellitosis were exhibited in the brain sample of one subject of group 2 and granulomatous meningoencephalitis was displayed in one subject of group 3. All cases were at moderate multifocal injury level. Figure $4 \mathrm{H}$ displays the encephalic section of rabbit \#1 confirmed with E. cuniculi infestation, where it can be observed an inflammatory infiltrate as well as plasmatic cells surrounding blood vessels. The optical nerve exhibited lymphoplasmacytic-meningitis in one rabbit of groups 2 and 3 , also satellitosis and gliosis was observed in a rabbit from group 3, Figure 4l. Those pathologies could be linked again to parasitic infection. No damage was observed in groups 1 and 4 either in brain or optical nerve (38-40).

Our animals were certified as healthy by the provider at the beginning of the study, however we detected infestation in our test population.Encephalitozoon cuniculi is a common opportunistic protozoan in laboratory animals which is very hard to identify until symptoms appear (41). It is an obligate intracellular microsporidian parasite whose target organs are kidney, lens and nervous tissue. Immunocompetent animals usually are subclinical carriers, but immunocompromised hosts often present chronic granulomatous inflammation. (42). Regarding this, we could hypothesize that the presence of this infection could have been associated and promoted by immunosuppressive activity of rapamycin, however no microorganisms could be detected in the subjects from groups 1 and 2, administered with rapamycin loaded formulations. As discussed above, parasitic infestation was confirmed only for rabbit \#1, administered with $450 \mu \mathrm{L}$ of liposomal vehicle. Also, all kinds of lesions encountered were statistically similar in all the treatment groups. It has been reported that cerebral lesions can only be observed about 8 weeks after initiation of antibody response to the infestation which takes place within 3 weeks post-infection (43). The duration of our experiment was of 3 weeks, with 10 additional days of quarantine where no obvious clinical signs of disease were observed. Since the age of the animals was of 10 weeks at the beginning of the quarantine period, is probably that these subjects were subclinical carriers that represented an infection focus of dissemination to the rest of the animals from urine excretion of spores that starts 3 to 5 weeks after antibody response (43). Thus, the rest of the animals were probably in a primary stage of the infestation at the moment of the necropsy due to urinary horizontal dissemination at quarantine period. This is also consistent with the presence of kidney injury in 8 of 12 subjects, considering that kidney damage is part of the primary stage of the disease. (44) 
Despite the infestation found in one subject, there was no relationship between injuries and treatment according to ANOVA analysis, Figure 5B, which means no effect can be attributable to rapamycin formulations. All the lesions observed were attributable to encephalitozoonosis and no other significant lesions were assessed in the high or low dose test groups. The fact that the confirmed subject was administered with liposomal vehicle without rapamycin suggests that other variable may have participated in the immunosuppression of the animals. In this manner, it has been studied that in assays with live laboratory animals, alterations due to stress often occur during toxicity studies and may interfere with the interpretation of the results. As discussed before, some of the evaluated parameters suggested that the animals were affected by stress during the study which may explain the development of the opportunistic infection. (45)

Finally, it is worth mentioning that the etiopathological origin ofEncephalitozoon Cuniculi in rabbit lens has been reported in literature (46). Ingestion of contaminated food or water is the most likely origin. Transplacental and respiratory routes are also possible however less likely due to certification of health from the animal provider. We also performed cultivation of the formulation, yielding negative results to this parasite.

\subsubsection{Biochemical assay}

Samples were analyzed to observe any renal or hepatic alteration caused by administration of liposomal formulations with respect to water for injection as a control. Differences in the aforementioned parameters were analyzed at 0,10 and 22 days after subconjunctival injection.

Biochemical measured values were between reference levels, therefore no influence from product administration can be assumed. Differences observed in urea and creatinine levels showing an upward trend in time can be considered normal due to increase in body weight of the rabbits due to normal growth. Cholesterol levels were significantly higher for group 3, treated with placebo liposomes. This difference was found because basal measurements in this group presented the highest values for this analyte therefore differences that cannot be associated with product administration. According to literature, administration of rapamycin has been associated with cholesterol serum elevation at rapamycin doses from 1-7 mg/day (47), however there was no increase observed in cholesterol levels in rapamycin treated groups. This could be explained by the very low dose that was locally administered, in conjunction with the liposomal carrier that encapsulates the drug and may inhibit systemic effects (48).

Concerning liver function tests, ALAT results showed difference between groups 1 and 2 for all sampling times because group 1 presented higher values for this parameter since basal measurements. Moreover, since the difference was observed throughout the whole study, it is probably not related to hepatic injury. This is also supported by a lack of difference between treatments for ASAT and AP results, which reaffirms that there is no evidence of damage on hepatocellular integrity.

In a similar manner, there were differences between group 1 and 3 in serum total protein results. Since these findings were observed since basal measurements it lacks significance considering that mean results of each group are in the normal range. These results presented a statistically significant gradual increment between sampling times, associated with normal animal growth. The same upward trend was observed for albumin and calcium, whereas for phosphorous there was observed a downward trend.

Total bilirubin concentration was significantly decreased from basal measurement with respect to second sampling time, but this was considered irrelevant because mean results of each sampling time were in reference range. Also, alkaline phosphatase results did not show alterations, therefore concluding that biliary flux was not affected. None of the serum biochemical parameters related to liver and kidney function showed results indicative of toxicity due to product administration in low or high dose, as well as the liposomal carrier.

Hematologic parameters showed significant differences in hematocrit and erythrocyte count between groups. This difference was due to higher values of group 4, presented in basal measurements. Bearing in mind that this difference was found in basal measurements from the control group, it has no physiological relevance. This may be explained as an effect secondary to a hemoconcentration state of the animals at the beginning of the study. Erythrocyte count was slightly out of normal range in basal measurement of the control group, 
along with hematocrit results that were in the upper limit of the normal range.

Significant differences in leucocyte count were also observed. Specifically, there was an increase in neutrophils in groups 1, 2 and 3. In all groups, an increase in monocytes and a decrease in lymphocytes at the first sampling time was observed. Even though statistically significant differences were observed, measurements were still in normal range. It has been reported that during toxicity studies with live laboratory animals, stress can affect diverse parameters including hematological assays. These results also suggested the possibility of an acute sub-clinical infectious process in all groups that will be discussed later. Some of the most common stress-related findings are lymphocyte depletion in thymus and spleen; resulting in altered circulating leukocyte counts, including increased neutrophils with decreased lymphocytes. (45) Animal stress is an inherent feature ofin vivo studies and may be triggered by many circumstances, among them, the handling process for dosing. In this case, the subconjunctival administration is a specialized type of injection that requires ocular topical anesthesia and must be performed by a specialist trained for this kind of administration. This process could have been more stressful for the animals than expected, summed with the expected stress generated by the sampling processes for biochemical and hematological parameters. This is consistent with the fact that lymphocytes and monocytes count alterations were also observed in the control group that was handled in the same manner, table 7.

\subsection{Evaluation of acute retinal toxicity in vivo in New Zealand rabbits by intravitreal injection.}

Intravitreal injection of rapamycin has shown good tolerability in some animal models so far $[9,10,14,15]$. However, the toxicity evaluation of every new formulation is mandatory being that the excipients of the formulation are not always compatible with the intraocular route (49). In this study, no statistically significant retinal acute toxicity was observed in electrical function evaluation or histological tissue examination. This is consistent with previous efforts to assess intravitreal toxicity with this drug. Fundus pictures showed no evident macroscopic alteration in retinal structure, however traumatic cataract formation was detected in some subjects 3 days after intravitreal administration. By slit-lamp examination, cataract formation was attributed to accidental lens traumatism during intravitreal injection technique since no trend or correlation could be determined between rapamycin amount and lens damage.

\subsubsection{Electroretinography}

Retinal damage can lead to vision loss due to lack of transmission of visual signal and has been in the top 5 most important causes of new drug candidate dismissal during drug development process (50). Dark-adapted ERG was used as a non-invasive in vivo evaluation of the electrical response of retinal cells. A significant decrease in amplitude of a or b-waves and/or prolongation of implicit times of these waves are indicative of retinal toxicity. In our study no statistically significant $(\mathrm{p}<0.05)$ reduction in amplitude, increased implicit time or alteration in waveform was observed between the basal measurements and post injection response. The same lack of significant difference was observed when comparing ERG results of tests groups versus control group 7 days after IVT administration.

This results are of great importance because we are challenging a new formulation of a drug that has been previously proved as safe for intravitreal injection by many other independent research groups, but also, this same drug has also been found to be toxic to the ocular structures when formulated in certain excipients with specific deterioration of retinal function evidenced by unfavorable electroretinography results (50). Interestingly, the b-wave response in the ERG of the group administered with $40 \mu \mathrm{g}$ of rapamycin was statistically different from control group and of its original basal measurements due to increased amplitude observed in this group after treatment. These may suggest a potential mechanism to improve impaired visual signaling in the retina. This amplitude augmenting effect has been previously reported at least once to our knowledge by De Paivaet al (2019), with the use of sustained-release rapamycin systems and was considered transient and clinically irrelevant (52). Also, a protective effect of rapamycin on visual impairment during inflammation has been reported as the attenuation in a- and b-wave reduction due to induced inflammation (53). Further studies to explore its effects on human retinal signal transmission would be interesting.

3.6.2. Clinical evaluation and histopathologic analysis 
Light microscopy showed normal tissue organization and cellularity of retinas in most subjects. Although, non-statistically significant alterations were observed, some subjects showed mild to severe injuries, specifically tissue degeneration and hyperplasia. Also, as can be appreciated in Fig. 11vi, lens fragmentation and presence of Morgagnian globules due to vacuole formation. This was consistent with slit-lamp examination and is probably related to histologic alterations observed since those subjects with cataract formation presented higher levels of histologic injury. In fact, it is noteworthy that the most severe injuries were observed in subjects treated with the intermediate dose of rapamycin, while only mild lesions were observed at the highest rapamycin dose. Despite these not significant $(\mathrm{p}<0.05)$ injuries, functionality of retinas was conserved from 40 to $440 \mu \mathrm{g}$ of liposomal rapamycin as proved by ERG evaluations.

Limitations of this study include the small number of eyes included in each experiment and unexpected development of a subclinical disease common in laboratory animals. Further research in order to elucidate intraocular pharmacokinetics of the formulation and ocular biodistribution of the drug will be determining to achieve aim of clinical testing for the treatment of eye immune mediate diseases. A $40 \mu \mathrm{g}$ liposomal rapamycin dose appears to have the best toxicity profile to be used by intraocular route, further research of its clinical effectivity is warranted.

\section{CONCLUSIONS}

Upon detailed examination of the formulation, no toxic effect was found in the different trials. Counting of micronucleus was normal, and the overall irritation score showed no significant differences when compared with the negative control. No presence of rapamycin in peripheral blood was found and no genotoxic damage was noted. Furthermore, the different histological, metabolic, biochemical and hematological evaluations demonstrated no rapamycin-related harmful effects after subconjunctival injection. Also, conservation of retina function after intravitreal injection was demonstrated. The liposome-encapsulated rapamycin formulation challenged in this article is safe to use in a dose range from 40 to $440 \mu \mathrm{g} / \mathrm{eye}$ in the different animal models experimented by subconjunctival or intravitreal injection. Furthermore, it shows great potential to be proven in a human clinical trial.

\section{ACKNOWLEDGEMENTS}

Part of this work was supported by CONACyT's Innovation Stimulus Program under grant 231008.

\section{REFERENCES}

(1) Neuhaus P, Klupp J, Langrehr J M. mTOR Inhibitors: An Overview. Liver Transpl. 2001;7(6):473-84. https://doi.org/10.1053/jlts.2001.24645

(2) Shen L-J, Lin Wu F-L. Nanomedicines in renal transplant rejection- focus on sirolimus. Int. J Nanomedicine, 2007;2(1):25-32. https://doi.org/10.2147/nano.2007.2.1.25

(3) Mahalati K, Kahan BD. Clinical Pharmacokinetics of sirolimus. Clin. Pharmacokinet. 2001;40:573-585. https://doi.org/10.2165/00003088-200140080-00002

(4) Simamora P, Alvarez J M, Yalkowsky S H. Solubilization of Rapamycin.Int J Pharm. 2001;213(1-2):25-9. https://doi.org/10.1016/s0378-5173(00)00617-7

(5) Sun M, Si L, Zhai X, et.al. The Influence of Co-Solvents on the Stability and Bioavailability of Rapamycin Formulated in Self-Microemulsifying Drug Delivery Systems. Drug Dev Ind Pharm.2011;37(8):98694. https://doi.org/10.3109/03639045.2011.553618

(6) Grinfield A A, Caufield C E, Schiksnis R A, Mattes J F, Chan K W. Acid Catalyzed Functionalization of rapamycin. Tetrahedron Lett.1994;35(37):6835-6838. https://doi.org/10.1016/0040-4039(94)85017-8

(7) Buech G, Bertelmann E, Pleyer U, Siebenbrodt I, Borchert H-H.J Ocul Pharmacol Ther. 2007;23(3):292303. https://doi.org/10.1089/jop.2006.130 
(8) Lloberas N, Torras J, Aplerovich G, Cruzado J M, et.al. Different renal toxicity profiles in the association of cyclosporine and tacrolimus with sirolimus in rats. Nephrol Dial Transpl.2008;23(10):3111-3119. https://doi.org/10.1093/ndt/gfn223

(9) Nguyen Q D, Ibrahim M A, Watters A, et.al. Ocular Tolerability and Efficacy of Intravitreal and Subconjunctival Injections of Sirolimus in Patients With Non-Infectious Uveitis: Primary 6-month Results of the SAVE study. J Ophthalmic Inflamm Infect . 2013;3(1):32. https://doi.org/10.1186/1869-5760-3-32

(10) Dugel P U, Blumenkranz M S, Haller J A, et.al. A Randomized, Dose-Escalation Study of Subconjunctival and Intravitreal Injections of Sirolimus in Patients With Diabetic Macular Edema. J Ophtalmol.2012;119(1):124-31. https://doi.org/10.1016/j.ophtha.2011.07.034

(11) Bhatt N, Dalal M, Tucker W, Obiyor D, Nussenblatt R, Nida Sen H. Subconjunctival Sirolimus in the Treatment of Autoimmune Non-Necrotizing Anterior Scleritis: Results of a Phase I/II Clinical Trial. Am J Ophtalmol. 2015;159(3):601-6. https://doi.org/10.1016/j.ajo.2014.12.009

(12) Shah M, Edman M C, Janga S R, et.al. A Rapamycin-Binding Protein Polymer Nanoparticle Shows Potent Therapeutic Activity in Supressing Autoimmune Dacryoadenitis in a Mouse Model of Sjogren's Syndrome.J Control Release. 2013;171(3):269-79. https://doi.org/10.1016/j.jconrel.2013.07.016

(13) Kahan B D. Sirolimus: A Comprehensive Review. Expert Opin Pharmacother. 2001;2(11):1903-17

(14) Douglas LC, Yi N Y, Davis J L, Salmon J H, Gilger B C, Ocular Toxicity and Distribuition of Subconjunctival and Intravitreal Rapamycin in Horses. J Vet Pharmacol Ther. 2008;31(6):511-6. https://doi.org/10.1111/j.1365-2885.2008.00986.x

(15) De Almeida Manzano R P, Peyman G A, Khan P, et.al. Testing Intravitreal Toxicity of Rapamycin in Rabbit Eyes. Arq Bras Oftalmol. 2009;72(1):18-22. https://doi.org/10.1590/s0004-27492009000100004

(16) WR Gombotz, B.5- Liposomes A2- Ratner, Buddy D, in: A.S Hoffman, F.J Schoen, J.E Lemons (Eds.), Biomaterials Science (Third Edition), Academic Press 2013, pp. 1039-1041.

(17) Patel P B, Shastri D H, Shelat P K, Shukla A K. Ophthalmic Drug Delivery System: Challenges and Approaches. Sys Rev Pharm.2010;1(2):113-120.

(18) Pattni B S, Chupin V V, Torchilin V P. New developments in Liposomal Drug Delivery. Chem Rev . 2015;115(19):10938-66. https://doi.org/10.1021/acs.chemrev.5b00046

(19) Kaur I P, Garg A, Singla A K, Aggarwal D. Vesicular Systems in Ocular Drug Delivery: An Overview. Int J Pharm. 2004;269(1):1-14. https://doi.org/10.1016/j.ijpharm.2003.09.016

(20) Meisner D, Mezei M. Liposome ocular delivery systems. Adv Drug Deliv Rev. 1995;16(1):75-93. https://doi.org/10.1016/0169-409X(95)00016-Z

(21) Bourlais C L, Acar , Zia H, Sado P A, Needham T, Leverge R. Ophthalmic Drug Delivery SystemsRecent Advances. Prog Retin Eye Res. 1998;17(1):33-58. https://doi.org/10.1016/s1350-9462(97)00002-5

(22) Barza M, Baum J, Tremblay C, Szoka F, D’Amico D J. Ocular Toxicity of Intravitreally Injected Liposomal Amphotericin B in Rhesus Monkeys. Am J Ophtlamol. 1985;100(2):259-63. https://doi.org/10.1016/00029394(85)90791-3

23) M R Mozafari, C J Reed, C Rostron. Cytotoxicity evaluation of anionic nanoliposomes and nanolipoplexes prepared by the heating method without employing volatile solvents and detergents." Pharmazie2007;62(3):205-9. PMID: 17416197

(24) Linares-Alba M A, Gómez-Guajardo M B, Furtado-Fonzar J, Brooks D E, García-Sánchez G A, BernadBernad M J. Preformulation Studies of a Liposomal Formulation Containing Sirolimus for the Treatment of Dry Eye Disease. J Ocul Pharmacol Ther.2016;32(1):11-22.

https://doi.org/10.1089/jop.2015.0032 
(25) García-Sánchez G A, \& Bernard M J B. 2017. U.S Patent No. 9,801,862. Washington, DC: U.S. Patent and Trademark Office.

26) Murilo-Bautista A, Nogura-Louzada R, Crunivel Isaac D L, et.al. In vivo and in vitro toxicity evaluation of liposome-encapsulated sirolimus. Int. J. Retin. Vitr. 2019; 5(35).

(27) OECD, Test No. 474 Mammalian Erithrocyte Micronucleus Test, OECD Publishing, 2014

(28) Batista-Duharte A, Murillo G J, Pérez U M, et.al. The Hen's Egg Test on Chorioallantoic Membrane: An Alternative Assay for the Assesment of the Irritating Effect of Vaccine Adjuvants. Int $J$ Toxicol.2016;35(6):627-633. https://doi.org/10.1177/1091581816672187

(29) FEUM, Prueba de pirógenos, in: FEUM (Ed.), Suplemento de dispositivos médicos, Secretaría de Salud, México 2001.

(30) Demircigil G C, Aykanat B, Fidan K, et.al. Micronucleus Frequencies in Peripheral Blood Lymphocytes of Children With Chronic Kidney Disease.Mutagenesis . 2011;26(5):643-50. https://doi.org/10.1093/mutage/ger027

(31) Kempen J H, Gangaputra S, Helzlsouer K J. Long-term risk of malignancy among patients treated with immunosuppressive agents for ocular inflammation: A critical assesment of evidence. Am J Ophtalmol. 2008;146(6):802-12. https://doi.org/10.1016/j.ajo.2008.04.035

(32) Blagosklonny M V. Molecular Damage in Cancer: An Argument for mTOR-driven Aging. Aging (Albany $N Y$ ). 2011;3(12):1130-41. https://doi.org/10.18632/aging.100422

(33) Tavaszi J, Budai P. The Use of HET-CAM Test in Detecting Ocular Irritation. Commun Agric Apll Biol Sci. 2007;72(2):137-41.

(34) Parnham M J, Wetzig H. Toxicity Screening of Liposomes. Chem Phys Lipids. 1993:64(1-3):263-74. https://doi.org/10.1016/0009-3084(93)90070-j

(35) Food and Drug Administration Center for Veterinary Medicine (2008). Guidance for Industry: Target Animal Safety for Veterinary Pharmaceutical Products. VICH GL43.

(36) Shah M, Edman M C, Janga S R, et.al. Rapamycin Eye Drops Suppress Lacrimal Gland Inflammation In a Murine Model of Sjogren's Syndrome.Invest Ophthalmol Vis Sci. 2017;58(1):372-385. https://doi.org/10.1167/iovs.16-19159

(36) H.L. Ioachim, L.J. Medeiros, Ioachim's Lymph Node Pathology, Wolters Kluwer Health/Lippincott Williams \& Wilkins2009.

(37) S.W. Barthold, S.M. Griffey, D.H. Percy, Pathology of Laboratory Rodents and Rabbits, Wiley2016.

(38) Sunohara-Neilson J, Brash M, Carman S, Nagy E, Turner P V. Experimental Infection of New Zealand White Rabbits (Oryctolagus cuniculi) with Leporid herpesvirus 4. Comp Med.2013;63(5):422-430. PMID 24210019

(39) P.J. Manning, D.H. Ringler, C.E. Newcomer, The Biology of the Laboratory Rabbit, Elsevier Science2014.

(40) Erdine S, Yucel A, Ozyalçin, et.al. Neurotoxicity of Midazolam in the Rabbit. Pain. 1999;80(1-2):419-23. https://doi.org/10.1016/s0304-3959(98)00240-1

(41) Cox J C, Hamilton R C, Attwood H D. An Investigation of the Route and Progression of Encephailtozzon Cuniculi Infection in Adult Rabbits.J Protozool. 1979;26(2):260-5. https://doi.org/10.1111/j.15507408.1979.tb02776.x.

(42) Pellett, Sarah. "Encephalitozoon cuniculi in rabbits: an overview." Companion Animal 21.5 (2016): 300-305. 
(43) Kunzel F, Joachim A. Encephalitozoonosis in Rabbits.Parasitol Res. 2010;106(2):299-309. https://doi.org/10.1007/s00436-009-1679-3

(44) Cox J C, Gallichio H A. Serological and Histological Studies on Adult Rabbits With Recent, Naturally Acquired Encephalitozoonosis.Res Vet Sci. 1978;24(2):260-1. PMID:653124

(45) Everds N E, Snyder P W, Bailey K L. Interpreting Stress Responses During Routine Toxicity Studies: A Review of the Biology, Impact, and Assessment. Toxicol Pathol. 2013;41(4):560-614. https://doi.org/10.1177/0192623312466452

46) Özkan Ö, Alcigir ME. Subacute Stage of Encephalitozoon cuniculi Infection in Eye Lesions of Rabbit in Turkey. Iran J Parasitol . 2018;13(2):301-309.

(47) Morrisett J D, Abdel-Fattah G, Hoogeveen R, et.al. Effects of Sirolimus on Plasma Lipids, Lipoprotein Levels, and Fatty Acid Metabolism in Renal Transplant Patients. J Lipid Res.2002;43(8):1170-80. PMID:12177161

(48) Falke L L, van Vuuren S H, Kazazi-Hyseni F, et.al. Local Therapeutic Efficacy With Reduced Systemic Side Effects by Rapamycin-Loaded Subcapsular Microspheres. Biomaterials.2015;42:151-60. https://doi.org/10.1016/j.biomaterials.2014.11.042

(49) Aguirre S A, Gukasyan H J, Younis H S, Huang W. Safety Assessment of Formulation Vehicles Following Intravitreal Administration in Rabbits. Pharm Res. 2018;35(9):173. https://doi.org/10.1007/s11095-018$2450-1$

(50) Mochizuki K, Torisaki M, Kawasaki K. Retinal toxicity of antibiotics: Evaluation by electroretinogram. Doc Ophthalmol.1988;69:195-202

(51) Patel S, Barnett J M, Kim S J. Retinal Toxicity of Intravitreal Polyethylene Glycol 400. J Ocul Pharmacol Th. 2016;32(2). https://doi.org/10.1089/jop.2015.0069

(52) Brandao-De Paiva M R, Almeida-Lage N, Andrade M C, et.al. Toxicity and in Vivo Release Profile of Sirolimus From Implants Into the Vitreous of Rabbits'Eyes. Doc Ophthalmol. 2019;138(1):3-19. https://doi.org/10.1007/s10633-018-9664-8

(53) Okamoto T, Ozawa Y, Kamoshita M. The Neuroprotective Effect of Rapamycin as a Modulator of the mTOR-NF- $x$ B Axis During Retinal Inflammation. PLoS One. 2016;11(1):e0146517. https://doi.org/10.1371/journal.pone.0146517.

\section{Hosted file}

TABLES AND FIGURES.docx available at https://authorea.com/users/360511/articles/482186toxicity-evaluation-of-a-novel-rapamycin-liposomal-formulation-after-subconjunctivaland-intravitreal-injection 